\title{
A placebo-controlled trial of Korean red ginseng extract for preventing Influenza-like illness in healthy adults
}

\author{
Ki-Chan Ha ${ }^{1,2 \dagger}$, Min-Gul Kim ${ }^{1 \dagger}$, Mi-Ra Oh${ }^{1}$, Eun-Kyung Choi ${ }^{1}$, Hyang-Im Back ${ }^{1,2}$, Sun-Young Kim ${ }^{1,2}$, Eun-Ok Park ${ }^{1,2}$, \\ Dae-Young Kwon ${ }^{4}$, Hye-Jeong Yang ${ }^{4}$, Min-Jeong Kim ${ }^{4}$, Hee-Joo Kang ${ }^{4}$, Ju-Hyung Lee ${ }^{5}$, Kyung-Min Choi ${ }^{6}$, \\ Soo-Wan Chae ${ }^{1,2}$ and Chang-Seop Lee (1,3* $^{*}$
}

\begin{abstract}
s
Background: Standardized Korean red ginseng extract has become the best-selling influenza-like illness (ILI) remedy in Korea, yet much controversy regarding the efficacy of the Korean red ginseng (KRG) in reducing ILI incidence remains. The aim of the study is to assess the efficacy of the KRG extract on the ILI incidence in healthy adults.

Methods/Design: We will conduct a randomized, double-blind, placebo-controlled study at the onset of the influenza seasons. A total of 100 subjects 30-70 years of age will be recruited from the general populations. The subjects will be instructed to take 9 capsules per day of either the KRG extract or a placebo for a period of 3 months. The primary outcome measure is to assess the frequency of ILI onset in participated subjects. Secondary variable measures will be included severity and duration of ILI symptoms. The ILI symptoms will be scored by subjects using a 4-point scale.

Discussion: This study is a randomized placebo controlled trial to evaluate the efficacy of the KRG extract compared to placebo and will be provided valuable new information about the clinical and physiological effects of the KRG extract on reduction of ILI incidence including flu and upper respiratory tract infections. The study has been pragmatically designed to ensure that the study findings can be implemented into clinical practice if KRG extract can be shown to be an effective reduction strategy in ILI incidence.
\end{abstract}

Trial Registration: NCT01478009.

\section{Background}

Respiratory viruses are a major cause of influenza-like illness(ILI) symptoms in children and adults, leading to substantial morbidity and mortality each year [1-5]. The complications of ILI symptoms may occur in young children $(<1$ year old) and elderly people $(>65$ years old), even though ILI symptoms is most often self-limited and restrained to the upper respiratory tract [6-8]. The ILI symptoms is characterized by sudden onset of symptoms such as high fever $\left(>38^{\circ} \mathrm{C}\right)$ and cough in the absence of other diagnosis $[9,10]$. Other symptoms

\footnotetext{
* Correspondence: Icsmd@jbnu.ac.kr

† Contributed equally

${ }^{1}$ Clinical Trial Center for Functional Foods of Chonbuk National University Hospital, Geumam-dong, Deokjin-gu, Jeonju 561-712, Republic of Korea
} Full list of author information is available at the end of the article including myalgia, headache, chills and fatigue can only be used as optional inclusion criteria. Although it is known that rhinovirus infections cause $10 \%$ to $40 \%$ of the upper respiratory tract infection [11], with coronavirus, parainfluenza virus, adenovirus, echovirus, and coxsackievirus accounting for the remainder of cases $[12,13]$, these viruses produce clinically indistinguishable disease, making specific viral diagnosis difficult $[14,15]$.

Many patients use complementary and alternative medicine (CAM) therapies to treat and prevent the acute respiratory illness in the worldwide. Moreover, few physicians are familiar with their efficacy or safety. Ginseng has been traditionally used in Asia for thousands of years to treat a variety of ailments. The putative active compounds derived from ginseng root processing

\section{() Biomed Central}


are the ginsenosides (saponin glycosides), and the watersoluble poly and oligosaccharides [16]. Various studies have shown that both families of compounds can modulate various parameters of the immune response in vitro and in vivo. In clinical trials, healthy subjects that consumed a standardized ginseng extract had a lower incidence of influenza and colds, higher antibody titers, and higher natural killer cell activity [17], as well as increased numbers of total lymphocytes and $\mathrm{T}$ helper cells [18]. Ginseng polysaccharide preparations increased cytokine production and mRNA expression by murine macrophages and spleen cells in vitro [19-21].

Currently, the ginseng was well known effect for improving the quality-of-life and immunomodulating effects [21-24]. However, there has been no data on the Korean red ginseng (KRG) accumulated for its preventive activity against ILI onset, as it has shown in American ginseng. The purpose of this study is to evaluate the efficacy of the KRG extract in reducing the frequency, severity and duration of ILI symptoms in healthy adults.

\section{Methods/Design}

\section{Study objectives}

The objectives of this RCT are to study whether the concentrated KRG extract can reduce the frequency, severity, and duration of ILI symptoms in healthy subjects.

\section{Primary objective}

To evaluate the efficacy of the KRG extract on the frequency of ILI onset in healthy subjects after 12 weeks of consumption.

\section{Secondary objectives}

To evaluate the following factors in healthy subjects after 12 weeks of consumption on:

\section{A) severity of ILI symptoms}

B) duration of ILI symptoms

\section{Ethics}

The study protocol and the written informed consent were approved by the Functional Foods Institutional Review Board of Chonbuk National University Hospital (CUH IRB 2010-02-016). Each subject will be notified regarding the study protocol. Written informed consent will be obtained from each subject.

\section{Study/trial design}

This study is a randomized, double-blind, placebo-controlled, two-armed parallel clinical trial, comparing the KRG extract to placebo. The design of the study will integrate rigorous in accord with principles set out in the Declaration of Helsinki and the Good Clinical Practice guidelines. Our study plan is summarized in Figure 1.

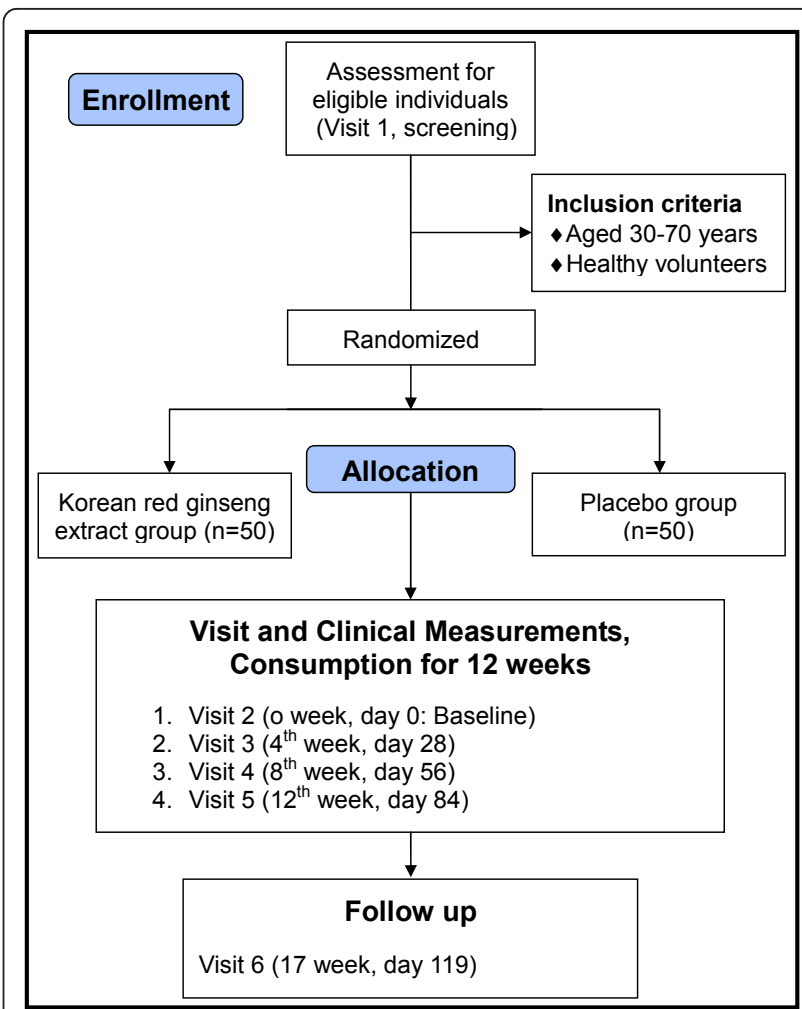

Figure 1 Flow diagram for study. Summary of the study flow.

Inclusion criteria for participation in the trial Inclusion criteria

Subjects will be included if they meet the following criteria:

A) Healthy volunteers aged 30 to 70 years

B) Ability to give informed consent

\section{Exclusion criteria}

The exclusion criteria for the study were:

A) Influenza vaccinated subjects

B) HIV infected and cancer patients

C) Cardiovascular disease, neurologic or psychiatric disease, renal, pulmonary and hepatic abnormalities

D) Upper respiratory infection within two weeks before study

E) History of disease that could interfere with the test products or impede their absorption, such as gastrointestinal disease (Crohn's Disease) or gastrointestinal surgery (a caesum or enterocele surgery are included)

F) Taking medications such as immunosuppressive drugs, corticosteroids, warfarin, phenalzine, pentobarbital, haloperidol, cyclosporine

G) Allergic or hypersensitive to ginseng 
H) Laboratory test, medical or psychological conditions deemed by the investigators to interfere with successful participation in the study

I) History of drug or alcohol abuse in prior 2 months

\section{Recruitment}

100 subjects will be recruited through local advertising and doctor referrals from hospital outpatients and general practice clinics. Interested subjects can telephone or email the trial coordinators at the trial conducting sites for further information. Subject information and consent forms will be sent to interested individuals to read over prior to scheduling their first visit.

\section{Randomization}

After enrollment, subjects will be randomly assigned to one of the two groups, either the KRG group or placebo group. The allocation ratio will be 1:1 in blocks of 2 . Randomization will be performed at a site remote from trial location. Random numbers will be generated by a computerized random-number generator through the block-randomization method of a software program (Excel, Microsoft Office 2007) for sequence generation. At the time of randomization subjects will draw an envelope. Each envelope contains a number that is concealed to the treatment allocation. Randomization sequence and allocation will be concealed to all study subjects, research staff, investigators and pharmacists until completion of the study. The allocation list will be protected by password access files and held by a noninvestigator independent. In the event of an emergency medical situation the individual's randomization code and group allocation can be identify.

\section{Outcome measures}

Subjects will be asked open-ended questions about frequency, severity, and duration of ILI symptoms during telephone follow-ups on days $1,28,57$, and 85 of treatment and days 35 post-treatment. Subjects will be also asked to report the study nurse immediately (within 24 hours), at the onset of ILI symptoms. Any unfavorable or unintended sign or symptom will be documented.

\section{Primary outcome}

The primary outcome is the frequency of ILI symptom onset. Subjects will be asked open-ended questions about frequency of ILI onset during study period. The frequency of ILI onset will be checked weekly via telephone.

\section{Secondary outcomes}

Secondary outcomes will be variety ILI symptoms (including fever, rhinorrhea, nasal congestion, sore throat, cough, sputum, dyspnea, diarrhea, mawkishness, vomiting, headache, and myalgia), severity of ILI symptoms, and duration of ILI symptoms. These outcomes will be checked weekly via telephone. The schedule of assessments is presented in Table 1.

\section{ILI assessments}

Subjects will be asked to record if they experienced any of the following 8 symptoms: sore throat, runny nose, nasal congestion, sneezing, hoarseness, myalgia, earaches, fever, headache, and cough (Table 2). In this selfassessment, subjects will be recorded the number of days they experienced each symptom. Subjects will be contacted by telephone each week to report the ILI symptoms and to assure compliance to the study protocol. They will be also asked to record if the study medication helped in shortening duration of their symptoms, and to list any additional medications taken for the symptoms. Subjects will be recorded daily symptom severity in a journal using a 4-point severity score index $(0=$ no symptom, $1=$ mild symptom, $2=$ moderate symptom and 3 = severe symptom).

\section{Statistical analysis \\ Baseline data and Outcomes data}

The effects of KRG extract or placebo treatment on the frequency, severity, and duration of symptoms related to ILI prior to the onset of influenza season will be analyzed. The Pearson chi-square test or Fisher's exact test (if the minimum expected count will be less than 2 or the ratio of expected counts lower than 5 will be more than $20 \%$ ) will be used to compare the proportions of subjects reporting symptoms related to ILI and adverse events. The data will be analyzed for all of the subjects who had been randomly assigned to a group, excluding those who provided only baseline information. An intention-to-treat and per-protocol analysis will be performed.

For the subset of subjects who reported ILI-related symptoms, the total score of severity and the duration for which symptoms will be reported and the two groups will be compared using Mann Whitney U test between two groups. A value of $\mathrm{p}<0.05$ will be considered statistically significant.

\section{Adverse events and monitoring safety}

All unexpected adverse events related to KRG intake will be reported to the investigator by subjects and write on the individual case report form by the investigator. Safety will be assessed by the reporting of clinical laboratory tests, vital sign measurements, and adverse events. Clinical laboratory tests, including AST/ALT, $\mathrm{BUN} /$ creatinine, red blood cell (RBC) count, white blood cell (WBC) count, hemoglobin, hematocrit, number of platelets, and number of differentiated cells will be determined at weeks 0 (baseline) and 12 (end of the trial). Vital signs of each subject will be checked with monitoring of adverse events (nausea/vomiting, fatigue, 
Table $1 \mathrm{~A}$ brief study schedule at every visit.

\begin{tabular}{|c|c|c|c|c|c|c|}
\hline & $\begin{array}{l}\text { Screening } \\
\text { Visit } 1\end{array}$ & $\begin{array}{c}\text { Baseline } \\
\text { Visit } 2\end{array}$ & Visit 3 & Visit 4 & Visit 5 & $\begin{array}{l}\text { Follow up } \\
\text { (Visit 6) }\end{array}$ \\
\hline & $\begin{array}{l}D-21 \\
\sim D-1\end{array}$ & $\begin{array}{l}\text { Week } 0 \\
\text { Do }\end{array}$ & $\begin{array}{l}\text { Week } 4 \\
\text { D28 }\end{array}$ & $\begin{array}{l}\text { Week } 8 \\
\text { D56 }\end{array}$ & $\begin{array}{l}\text { Week } 12 \\
\text { D84 }\end{array}$ & $\begin{array}{l}\text { Week } 17 \\
\text { D119 }\end{array}$ \\
\hline Informed consent form & O & & & & & \\
\hline Demographic information taking $^{1}$ & O & & & & & \\
\hline Medical history taking & ○ & ○ & O & O & $\circ$ & \\
\hline Inclusion/exclusion criteria check & O & O & & & & \\
\hline Physician examination $^{2}$ & O & & & & O & \\
\hline Vital sign measurement & O & O & O & O & O & \\
\hline Concomitant drugs check & O & O & O & O & 0 & \\
\hline Electrocardiogram(ECG) & O & & & & O & \\
\hline Questionnaires for symptoms related to ILI ${ }^{3}$ & O & O & O & O & O & ○ \\
\hline Laboratory test ${ }^{4}$ & O & & & & o & \\
\hline Study product distribution & & ○ & ○ & ○ & & \\
\hline Compliance checking & & & O & O & ○ & \\
\hline Adverse event monitoring & & & O & ○ & ○ & O \\
\hline \multicolumn{7}{|l|}{${ }^{\circ}$ : Item need to be performed during the visit } \\
\hline \multicolumn{7}{|c|}{${ }^{1}$ Sex, date of birth, age, contact address and phone number } \\
\hline \multicolumn{7}{|c|}{2 Include present illness, past history taking } \\
\hline \multicolumn{7}{|c|}{${ }^{3}$ Frequency of ILI symptom onset, severity of ILI symptoms, and duration of ILI symptoms } \\
\hline \multicolumn{7}{|c|}{$\begin{array}{l}{ }^{4} \text { Blood test (WBC, RBC, hemoglobin, hematocrit, platelets count, AST, ALT, ALP, gamma-GTP, albumin, BUN, creatinine, glucose, total bilirubin, total protein), urine te } \\
\text { (specific gravity, } p H \text {, protein, ketone, glucose, bilirubin, urobilinogen, nitrite, microscopic (RBC,WBC)) }\end{array}$} \\
\hline
\end{tabular}

allergic reaction, and any adverse events related to KRG extract intake) after each visit.

\section{Compliance}

The KRG soft capsules remaining after each visit will be quantified in order to enhance medication compliance. Subjects whose compliance with the KRG extract capsules is $\leq 70 \%$ of the total dose will be considered to have dropped-out.

\section{Sample size}

This pilot study is the first clinical trials using KRG extract for Korean population. So, there was limited

Table 2 Questionnaire for Influenza-Like IIIness

\begin{tabular}{|c|c|c|c|}
\hline Symptoms & Score & Duration (days) & $\begin{array}{c}\text { nonattendance } \\
\text { (days) }\end{array}$ \\
\hline Sore throat & $\square 0 \square 1 \square 2 \square 3$ & & \\
\hline Runny nose & $\square 0 \square 1 \square 2 \square 3$ & & \\
\hline Nasal congestion & $\square 0 \square 1 \square 2 \square 3$ & & \\
\hline Sneezing & $\square 0 \square 1 \square 2 \square 3$ & & \\
\hline Hoarseness & $\square 0 \square 1 \square 2 \square 3$ & & \\
\hline Myalgia & $\square 0 \square 1 \square 2 \square 3$ & & \\
\hline Earaches & $\square 0 \square 1 \square 2 \square 3$ & & \\
\hline Fever & $\square 0 \square 1 \square 2 \square 3$ & & \\
\hline Headache & $\square 0 \square 1 \square 2 \square 3$ & & \\
\hline Cough & $\square 0 \square 1 \square 2 \square 3$ & & \\
\hline Total score & & & \\
\hline
\end{tabular}

information about the prevention of influenza incidence or symptom releases. Therefore, we had designed a preliminary test using total 100 participants (50:50).

\section{Discussion}

In this study, we will examine whether the KRG extract administration can significantly reduce the frequency, severity, and duration of ILI symptoms. A 1000-mg dose of KRG will be taken 3 times daily by subjects for 12 weeks. Initial ILI symptoms will be confirmed by the collaborating physician at the screening visit of selfreported ILI symptoms, and relief of symptoms will be confirmed within 7 day of the subject's self-reported recovery. Subjects will be recorded daily symptom severity in a journal using a 4-point severity score.

The aim of this study is to evaluate the efficacy of the KRG extract on the frequency, severity, and duration of ILI symptoms. For this purpose, the ability of the KRG extract to reduce the ILI incidence will be assessed in 100 healthy volunteers.

In this study, we expect that the KRG extract can effectively reduce the ILI incidence. Some studies have assessed the relative potency of ginseng extracts by evaluating their ability to prevent the upper respiratory tract infections. The ginseng extracts has been studied in 3 recent RCTs to prevent the common cold, the flu, or upper respiratory infections. Two studies did not show differences between the ginseng extract and placebo in 
terms of decreasing duration, severity, or frequency of overall symptoms $[25,26]$. One study demonstrated that the ginseng extracts decreased duration, severity, and frequency of symptoms [27].

To the best of our knowledge, there has been no report demonstrating the preventive efficacy of KRG extract on ILI incidence. Therefore, if this study will be successfully performed, the KRG extract may offer beneficial effects in reducing ILI incidence. And these results will be applied to children and elderly populations. To draw confirmative conclusion about the preventive efficacy and safety of the KRG extract on ILI incidence, a full-scale RCT is being conducted now. If there are any conclusions in the near future, we would like to publish them later.

\section{Acknowledgements}

This study was financially supported by a contract with Ministry for Food, Agriculture, Forestry and Fisheries (MIFAFF)/Korea Food Research Institute (KFRI)/Institute of Jinan Red Ginseng (IJRG) and is being conducted at the Clinical Trial Center for Functional Food of Chonbuk National University Hospital.

\section{Author details}

${ }^{1}$ Clinical Trial Center for Functional Foods of Chonbuk National University Hospital, Geumam-dong, Deokjin-gu, Jeonju 561-712, Republic of Korea. ${ }^{2}$ Department of Medical Nutrition Therapy, Chonbuk National University Medical School, Geumam-dong, Deokjin-gu, Jeonju 561-180, Republic of Korea. ${ }^{3}$ Department of Internal Medicine, Chonbuk National University Medical School, Geumam-dong, Deokjin-gu, Jeonju 561-180, Republic of Korea. ${ }^{4}$ Korea Food Research Institute, Baekhyeon-dong, Bundang-gu, Seongnam-City 463-746, Gyeonggi-do, Republic of Korea. ${ }^{5}$ Department of Preventive Medicine, Chonbuk National University Medical School, Geumamdong, Deokjin-gu, Jeonju 561-180, Republic of Korea. ${ }^{6}$ Institute of Jinan Red Ginseng, Banwol-ri, Jinan-gun 657-801, Republic of Korea.

\section{Authors' contributions}

DYK, SWC, KMC, KCH, MGK, and CSL received the research funding, led the entire study, and drafted the manuscript. MRO, EKC, HIB, SYK, EOP, JHL, HJY, $\mathrm{MJK}$, and HJK participated in the design of the study and will be performed the statistical analysis.

All authors read and approved the final manuscript.

\section{Competing interests}

The authors declare that they have no competing interests.

Received: 12 January 2012 Accepted: 8 February 2012

Published: 8 February 2012

\section{References}

1. Kaye M, Skidmore S, Osman H, Weinbren M, Warren R: Surveillance of respiratory virus infections in adult hospital admissions using rapid methods. Epidemiol Infect 2006, 134(4):792-798.

2. Jansen AG, Sanders EA, Hoes AW, van Loon AM, Hak E: Influenza- and respiratory syncytial virus-associated mortality and hospitalisations. Eur Respir J 2007, 30(6):1158-1166.

3. Camps M, Ricart S, Dimova V, Rovira N, Munoz-Almagro C, Garcia JJ, PonsOdena M, Marcos MA, Pumarola T: Prevalence of human metapneumovirus among hospitalized children younger than 1 year in Catalonia, Spain. J Med Virol 2008, 80(8):1452-1460.

4. Johnson BF, Wilson LE, Ellis J, Elliot AJ, Barclay WS, Pebody RG, McMenamin J, Fleming DM, Zambon MC: Fatal cases of influenza a in childhood. PLoS One 2009, 4(10):e7671.
5. Singleton RJ, Bulkow LR, Miernyk K, DeByle C, Pruitt L, Hummel KB, Bruden D, Englund JA, Anderson LJ, Lucher L, et al: Viral respiratory infections in hospitalized and community control children in Alaska. J Med Virol 2010, 82(7):1282-1290.

6. Hall CB: Hospital-acquired pneumonia in children: the role of respiratory viruses. Semin Respir Infect 1987, 2(1):48-56.

7. Flamaing J, Engelmann I, Joosten E, Van Ranst M, Verhaegen J, Peetermans WE: Viral lower respiratory tract infection in the elderly: a prospective in-hospital study. Eur J Clin Microbiol Infect Dis 2003, 22(12):720-725.

8. Cilla G, Onate E, Perez-Yarza EG, Montes M, Vicente D, Perez-Trallero E: Viruses in community-acquired pneumonia in children aged less than 3 years old: High rate of viral coinfection. J Med Virol 2008, 80(10):1843-1849.

9. Aguilera JF, Paget WJ, Mosnier A, Heijnen ML, Uphoff H, van der Velden J, Vega T, Watson JM: Heterogeneous case definitions used for the surveillance of influenza in Europe. Eur J Epidemiol 2003, 18(8):751-754.

10. Nichol KL: Heterogeneity of influenza case definitions and implications for interpreting and comparing study results. Vaccine 2006, 24(4446):6726-6728.

11. Kirkpatrick GL: The common cold. Prim Care 1996, 23(4):657-675.

12. Engel JP: Viral upper respiratory infections. Semin Respir Infect 1995, 10(1):3-13.

13. Lowenstein SR, Parrino TA: Management of the common cold. Adv Intern Med 1987, 32:207-233.

14. Tyrrell DA, Cohen S, Schlarb JE: Signs and symptoms in common colds. Epidemiol Infect 1993, 111(1):143-156.

15. Gwaltney JM Jr: Rhinovirus infection of the normal human airway. Am J Respir Crit Care Med 1995, 152(4 Pt 2):S36-39.

16. Tan BK, Vanitha J: Immunomodulatory and antimicrobial effects of some traditional chinese medicinal herbs: a review. Curr Med Chem 2004, 11(11):1423-1430.

17. Scaglione F, Cattaneo G, Alessandria M, Cogo R: Efficacy and safety of the standardised Ginseng extract G115 for potentiating vaccination against the influenza syndrome and protection against the common cold [corrected]. Drugs Exp Clin Res 1996, 22(2):65-72.

18. Scaglione F, Ferrara F, Dugnani S, Falchi M, Santoro G, Fraschini F: Immunomodulatory effects of two extracts of Panax ginseng C.A. Meyer. Drugs Exp Clin Res 1990, 16(10):537-542.

19. Lim DS, Bae KG, Jung IS, Kim CH, Yun YS, Song JY: Anti-septicaemic effect of polysaccharide from Panax ginseng by macrophage activation. $J$ Infect 2002, 45(1):32-38.

20. Song JY, Han SK, Son EH, Pyo SN, Yun YS, Yi SY: Induction of secretory and tumoricidal activities in peritoneal macrophages by ginsan. Int Immunopharmacol 2002, 2(7):857-865.

21. Kim KH, Lee YS, Jung IS, Park SY, Chung HY, Lee IR, Yun YS: Acidic polysaccharide from Panax ginseng, ginsan, induces Th1 cell and macrophage cytokines and generates LAK cells in synergy with rlL-2. Planta Med 1998, 64(2):110-115.

22. Wang $M$, Guilbert $L$, Ling L, Li J, Wu Y, Xu S, Pang P, Shan JJ: Immunomodulating activity of CVT-E002, a proprietary extract from North American ginseng (Panax quinquefolium). J Pharm Pharmacol 2001, 53(11):1515-1523.

23. Tomoda M, Hirabayashi K, Shimizu N, Gonda R, Ohara N, Takada K: Characterization of two novel polysaccharides having immunological activities from the root of Panax ginseng. Biol Pharm Bull 1993, 16(11):1087-1090.

24. Shin KS, Kiyohara H, Matsumoto T, Yamada H: Rhamnogalacturonan II from the leaves of Panax ginseng C.A. Meyer as a macrophage Fc receptor expression-enhancing polysaccharide. Carbohydr Res 1997, 300(3):239-249.

25. Vohra S, Johnston BC, Laycock KL, Midodzi WK, Dhunnoo I, Harris E, Baydala L: Safety and tolerability of North American ginseng extract in the treatment of pediatric upper respiratory tract infection: a phase II randomized, controlled trial of 2 dosing schedules. Pediatrics 2008, 122(2):e402-410

26. McElhaney JE, Goel V, Toane B, Hooten J, Shan JJ: Efficacy of COLD-fX in the prevention of respiratory symptoms in community-dwelling adults: a randomized, double-blinded, placebo controlled trial. J Altern Complement Med 2006, 12(2):153-157. 
27. Predy GN, Goel V, Lovlin R, Donner A, Stitt L, Basu TK: Efficacy of an extract of North American ginseng containing poly-furanosyl-pyranosylsaccharides for preventing upper respiratory tract infections: a randomized controlled trial. CMAJ 2005, 173(9):1043-1048.

Pre-publication history

The pre-publication history for this paper can be accessed here: http://www.biomedcentral.com/1472-6882/12/10/prepub

doi:10.1186/1472-6882-12-10

Cite this article as: Ha et al:: A placebo-controlled trial of Korean red ginseng extract for preventing Influenza-like illness in healthy adults.

BMC Complementary and Alternative Medicine 2012 12:10.

Submit your next manuscript to BioMed Central and take full advantage of:

- Convenient online submission

- Thorough peer review

- No space constraints or color figure charges

- Immediate publication on acceptance

- Inclusion in PubMed, CAS, Scopus and Google Scholar

- Research which is freely available for redistribution

Submit your manuscript at www.biomedcentral.com/submit 